\title{
EDUCAÇÃO EM SAÚDE NO SERVIÇO DE HEMODINÂMICA: UMA REVISÃO INTEGRATIVA
}

\section{Suellen Cristina da Silva Chaves}

Enfermeira, graduada em Enfermagem pela Faculdade Evangélica do Paraná. Residente do Programa em Cardiovascular da Universidade Federal do Paraná/ UFPR, Curitiba (PR), Brasil.

\section{Tatiana Brusamarello}

Enfermeira, Mestre e doutoranda em enfermagem pela Universidade Federal do Paraná. Enfermeira CHC/UFPR. Coordenadora Educação Continuada do Complexo Hospital de Clínicas Curitiba (PR), Brasil.

\section{Resi Rejane Huernermann}

Enfermeira, Membro da Sociedade Brasileira de Hemodinâmica e Cardiologia Intervencionista e Responsável pelo Serviço de Hemodinâmica do CHC/UFPR, Curitiba, Brasil.
RESUMO: Este artigo teve como objetivo conhecer as ações educativas em saúde realizadas no serviço de hemodinâmica acerca do exame de cineangiocoronariografia. Trata-se de uma revisão integrativa, realizada no mês de outubro de 2016, com análise de publicações indexadas nas bases de dados: LILACS, BDENF, SCOPUS e PubMed no período de 2011 e 2016. Foram selecionados oito estudos que correspondiam aos critérios de inclusão e exclusão, sendo divididos em dois temas principais: déficit de conhecimento dos pacientes sobre cineangiocoronariografia e a construção do conhecimento por meio de diferentes abordagens metodológicas. Os resultados ressaltam a escassez de estudos voltados para essa temática no Brasil e a necessidade do uso de novas tecnologias metodológicas como fator de consolidação da educação em saúde no serviço de hemodinâmica.

PALAVRAS-CHAVE: Educação em saúde; Doença na artéria coronariana; Cateterismo cardíaco; Hemodinâmica; Enfermagem cardiovascular.

\section{HEALTH EDUCATION WITHIN THE HEMODYNAMIC SERVICE: AN INTEGRATIVE REVIEW}

ABSTRACT: Current analysis aimed at acknowledging health educational activities in the hemodynamic service on the coronary angiography exam. The integrative review occurred in October 2016, with analysis of publications indexed in databases LILACS, BDENF, SCOPUS and PubMed between 2011 and 2016. Eight papers were selected following inclusion and exclusion criteria, divided into two main themes: knowledge deficit of patients on coronary angiography and the construction of knowledge by different methodological approaches. Results enhance the dearth of studies on the theme in Brazil and the need for new methodological technologies as a consolidation factor in health education in hemodynamics.

KEY WORDS: Health education; Coronary disease; Heart catheters; Hemodynamics; cardiovascular nursing.

\section{INTRODUÇÃO}

As doenças cardiovasculares são as protagonistas de aproximadamente $30 \%$ dos óbitos anuais no Brasil, isto corresponde a mais de 308 mil óbitos, sendo o infarto agudo do miocárdio (IAM)

Autor correspondente:

Suellen Cristina da Silva Chaves

E-mail: suellenchaves29@gmail.com e o acidente vascular cerebral (AVC) as principais causas, com maior prevalência no sexo masculino com idade entre 55 e 59 anos $^{1}$.

Baseado neste perfil epidemiológico, a Sociedade Brasileira 
de Cardiologia (SBC) desenvolveu, em sintonia com a proposta global da Organização Mundial de Saúde (OMS), a I Diretriz Nacional de Prevenção Cardiovascular, com o objetivo de reduzir até $25 \%$ dos óbitos por doenças não transmissíveis no mundo até o ano de $2025^{2}$.

Esta diretriz norteia e uniformiza ações de prevenção por meio de abordagem de fatores comportamentais de risco, como tabagismo, dietas não saudáveis, obesidade, sedentarismo e alcoolismo; enfatiza, ainda, a importância do diagnóstico e tratamento precoce, por meio de serviços de orientação ou administração adequada de medicamentos, para as pessoas que apresentem um ou mais fatores de risco como hipertensão, diabetes, dislipidemia ou doença já estabelecida ${ }^{2}$.

Dentreosmétodosutilizados paraodiagnósticode alterações estruturais, fisiológicas e de doenças cardíacas, destaca-se como padrão ouro a cineangiocoronariografia (cateterismo cardíaco), que, além de fornecer informações prognósticas que permitem o planejamento terapêutico adequado, também é uma intervenção menos invasiva e de menor custo, quando comparado a intervenções cirúrgicas $^{3,4}$. A cineangiocoronariografia é um exame de alta complexidade realizado no laboratório de hemodinâmica, por equipe multiprofissional de saúde, entre os quais, enfermeiros especialistas e profissionais de enfermagem do nível técnico5.

O exame consiste na introdução de um cateter que é avançado até a aorta e o ventrículo esquerdo, por intermédio de uma punção na artéria radial, braquial ou femoral. As imagens das câmaras cardíacas, valvas e das artérias coronárias são capturadas, após infusão de contraste iodado pelo cateter, por aparelho de raio-X e projetada em um monitor disposto na sala de hemodinâmica ${ }^{3,6}$.

Devido à simbologia do coração como órgão motor da vida, associado às incertezas e dúvidas a respeito do exame, ocorre a exacerbação de sentimentos como ansiedade, preocupações e estresse nos pacientes no período pré-procedimento ${ }^{3-6}$. Neste sentido, a realização de ações educativas em saúde, por parte da equipe de enfermagem nos laboratórios de hemodinâmica, acerca do exame cateterismo cardíaco torna-se fundamental, devendo ser realizada no momento em que se antecede o exame para que tais fatores sejam amenizados, contribuindo para a satisfação e qualidade no atendimento ${ }^{7}$.

A educação em saúde constitui em um conjunto de práticas pedagógicas de conteúdo técnico, político e científico com caráter participativo e emancipatório, devendo potencializar o empoderamento dos sujeitos para atuarem no enfrentamento de situações que interfiram na sua qualidade de vida ${ }^{8-10}$. Esta educação deve ser baseada em evidências científicas, de forma comprometida com os princípios da ética e da democratização do conhecimento?.

Deste modo, este estudo tem como questão norteadora: "Como são realizadas as ações de educação em saúde para usuários do serviço de hemodinâmica?" E como objetivo: conhecer as ações educativas em saúde, realizadas com usuários nos serviços de hemodinâmica acerca da cineangiocoronariografia. Acredita-se que o resultado deste estudo possa vir a contribuir para a melhoria da qualidade de assistência de enfermagem prestada nos serviços de hemodinâmica.

\section{METODOLOGIA}

O presente estudo trata-se de uma revisão integrativa. Método que proporciona a avaliação crítica e síntese das evidências disponíveis sobre o tema investigado com base em pesquisas já publicadas. Identificam-se tendências da produção e a incorporação da aplicabilidade dos resultados de estudos significativos na prática e apontam-se lacunas que direcionam para o desenvolvimento de futuras pesquisas, sempre pautadas na análise ampla e sistemática de estudos científicos ${ }^{11}$.

Foram realizadas seis etapas: I. Identificação do problema; II. Formulação da pergunta norteadora; III. Definição da amostragem, escolha dos descritores, base de dados para consulta; IV. Critérios de inclusão e exclusão; V. Extração de dados em bases científicas e seleção dos artigos; VI. Análise e síntese dos resultados ${ }^{11}$.

A pergunta que norteou este estudo, como citado anteriormente, foi "Como são realizadas as ações de educação em saúde para usuários do serviço de hemodinâmica?"

Para o levantamento dos artigos foi realizada 
uma busca nos sistemas de base de dados on-line: Base de Dados em Enfermagem (BDENF), Literatura LatinoAmericana em Ciências da Saúde (LILACS), National Library of Medicine (PubMed) e SCOPUS no mês de novembro de 2016. Para tanto, utilizou-se a seguinte estratégia de busca: Na BDENF e LILACS os descritores utilizados foram "educação em saúde", "doença na artéria coronariana", "enfermagem cardiovascular", "cateterismo cardíaco", e "hemodinâmica". No PubMed e SCOPUS os artigos foram identificados com os descritores: "bealth education", "coronary artery disease", "cardiovascular nursing", "catheterization cardiac" e "bemodynamics".

Para seleção dos periódicos foram utilizadas as expressões booleanas "AND" e "OR", com a combinação dos termos: "educação em saúde" AND ("doença na artéria coronariana" $O R$ "cateterismo cardíaco" $O R$ "hemodinâmica"). No idioma inglês, as combinações foram "bealth education" AND ("coronary artery disease" $O R$ "catheterization cardiac" OR "bemodynamics").

A seleção da amostra compreendeu os seguintes critérios de inclusão: ser artigo científico original disponível na íntegra eletronicamente, nos idiomas inglês, espanhol e português tendo como período de publicação os anos entre 2011 a 2016 e responder a questão de pesquisa. Foram excluídas teses, dissertações, artigos em duplicatas.

$\mathrm{Na}$ busca nas bases de dados foram encontrados 1.156 publicações, sendo três na base de dados BDENF, 74 na base de dados LILACS, 220 na PubMed e 859 na SCOPUS. Após leitura dos títulos e resumos foram selecionados 38 estudos. A partir desta seleção procedeuse à leitura destes na íntegra. A amostra final resultou em sete artigos. No Quadro 1 é apresentado o fluxograma do percurso metodológico da pesquisa nas bases de dados, baseado nos critérios do Preferred ReportingItems for Systematic Reviewand a Meta-Analyses (PRISMA) ${ }^{12}$.

Para a análise final dos resultados foi elaborado um instrumento que contempla os itens: revista, ano, categoria profissional, referências, título, objetivo, tipo do estudo e principais resultados. Posteriormente, os dados foram submetidos à análise crítica, descrição dos resultados e discussão.
Quadro 1. Distribuição dos artigos encontrados, excluídos e selecionados nas bases eletrônicas de dados

\begin{tabular}{|c|c|c|c|}
\hline 胥 & $\begin{array}{c}\text { Estudos identificados através da } \\
\text { busca em base de dados } \\
\mathrm{n}=1.156 \\
\text { Bdenf }=3 \\
\text { Lilacs }=74 \\
\text { PubMed }=220 \\
\text { SCOPUS }=859\end{array}$ & & \\
\hline \multirow{3}{*}{ 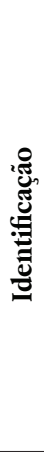 } & $\begin{array}{l}\text { Refinamento da busca consideran- } \\
\text { do os últimos cinco anos e idioma } \\
\text { inglês, espanhol e português n } \\
=171\end{array}$ & $\rightarrow$ & $\begin{array}{l}\text { Total de estudos excluí- } \\
\qquad \operatorname{dos} n=946\end{array}$ \\
\hline & $\begin{array}{l}\text { Total de estudo após remoção dos } \\
\text { duplicados } n=116\end{array}$ & $\rightarrow$ & $\begin{array}{l}\text { Estudos duplicados } \\
\text { excluídos } n=55\end{array}$ \\
\hline & $\begin{array}{c}\text { Estudos selecionados para leitura } \\
\text { de título e resumo } n=116\end{array}$ & $\rightarrow$ & $\begin{array}{l}\text { Estudos excluídos após } \\
\text { a leitura dos resumos } \mathrm{n} \\
=78 \text {; Indisp. }=01 ; \mathrm{NP} \\
=01 ; \mathrm{NA}=05 ; \mathrm{NQ} \text { ou } \\
\mathrm{NT}=71\end{array}$ \\
\hline 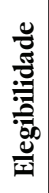 & $\begin{array}{c}\text { Leitura de artigos na íntegra para } \\
\text { elegibilidade } n=38\end{array}$ & $\rightarrow$ & $\begin{array}{l}\text { Artigos excluídos após } \\
\text { leitura na íntegra } \mathrm{n}= \\
07 \mathrm{NQ}=09 ; \mathrm{NT}=22\end{array}$ \\
\hline$\frac{\mathscr{O}}{\stackrel{0}{ }}$ & $\begin{array}{l}\text { Estudos incluídos na revisão } \mathrm{n}= \\
\begin{array}{c}07 \text { Bdenf }=01 \text { Lilacs }=02 \text { PubMed } \\
=01 \text { SCOPUS }=03\end{array}\end{array}$ & & \\
\hline
\end{tabular}

Fonte: A autora.

Legenda: $\mathrm{NA}=$ não é artigo; $\mathrm{NP}=$ não é pesquisa; $\mathrm{NQ}=$ não responde às questões; $\mathrm{NT}=$ não é da temática; Indisp. = Artigo indisponível eletronicamente na íntegra ${ }^{12}$.

\section{RESULTADOS}

Os sete artigos que compuseram esta revisão foram caracterizados quanto ao ano de publicação, país, área da saúde, objetivo e tipo de pesquisa, dispostos no Quadro 2. Observa-se que as publicações foram maiores no ano de 2013, num total de 42,8\% (n=3), já em 2016 com 28,5\% ( $n=2)$ dos artigos, seguido por 2011 e 2012 ambos com 14,3\% $(n=1)$ das publicações cada, em 2015 e 2014 não ocorreram publicações sobre a temática. Este número reduzido de manuscritos publicados demonstra que estudos relacionados à educação em saúde nos serviços de hemodinâmica ainda é incipiente.

Em relação ao país de publicação dos sete estudos, 42,8\% (n=3) são nacionais e 57,2\% (n=4) internacionais, todos desenvolvidos em ambientes hospitalares. Quanto à categoria profissional dos autores, considerando apenas o primeiro autor de cada produção, temos: enfermagem com $64,2 \%(n=4,5)$ e medicina com $35,8 \%(n=2,5)$ dos estudos. Os profissionais dessas categorias demonstram maiores preocupações quanto 
aos saberes referentes ao processo de educação em saúde, uma vez que nestas áreas há renovação frequente de toda a base normativa, exigindo a busca constante do conhecimento e qualidade no atendimento prestado. Além disso, trata-se de um tema que exige atuação ativa dos envolvidos, sendo inclusive, objeto de prevenção de agravos à saúde. A enfermagem destaca-se na educação em saúde por ser a profissão que atualmente procura aprimorar conhecimento para a melhoria no atendimento e humanização da assistência ${ }^{10}$.

As abordagens variam entre artigos de pesquisa qualitativa 28,5\% $(n=2)$, estudos de pesquisa coorte $28,5 \%$ $(n=2)$ e pesquisa transversal $42,9 \%(n=3)$. Quanto ao conteúdo disposto em cada artigo, verifica-se que todas as publicações $(n=7)$ apresentam os objetivos do estudo de forma clara, possibilitando o fácil entendimento do leitor.

Com esta revisão observa-se que os profissionais de enfermagem é uma das categorias que mais realizou publicações, demonstram maiores preocupações quanto aos saberes referentes à educação em saúde, e buscam constantemente o conhecimento e a qualidade nos serviços prestados (Quadro 2).

Quadro 2. Distribuição dos artigos selecionados

\begin{tabular}{|c|c|c|c|c|}
\hline $\begin{array}{l}\text { Ano } \\
\text { País }\end{array}$ & $\begin{array}{l}\text { Área da } \\
\text { saúde }\end{array}$ & Título & Objetivo & $\begin{array}{l}\text { Tipo de } \\
\text { Estudo }\end{array}$ \\
\hline $\begin{array}{l}2011 \\
\text { Brasill }\end{array}$ & Enfermagem & $\begin{array}{l}\text { Digital vídeodisc explicativo em pacientes } \\
\text { submetidos ao cateterismo cardíaco diag- } \\
\text { nóstico }\end{array}$ & $\begin{array}{l}\text { Avaliar o conhecimento dos pacientes pré-ca- } \\
\text { teterismo cardíaco com diagnóstico ambula- } \\
\text { torial, após digital vídeo disc explicativo }\end{array}$ & Transversal \\
\hline $\begin{array}{l}2012 \\
\text { Reino } \\
\text { Unido }\end{array}$ & Medicina & $\begin{array}{l}\text { Coronary Angioplasty and the Internet: } \\
\text { What Can Patients Searching Online Expect } \\
\text { to Find? }\end{array}$ & $\begin{array}{l}\text { Avaliar a qualidade da informação on-line } \\
\text { disponível sobre angioplastia coronária }\end{array}$ & Transversal \\
\hline $\begin{array}{l}2013 \\
\text { Brasil }\end{array}$ & Enfermagem & $\begin{array}{l}\text { Visão do enfermeiro frente à prática da edu- } \\
\text { cação em saúde no ambiente hospitalar }\end{array}$ & $\begin{array}{l}\text { Conhecer a visão do enfermeiro frente à } \\
\text { prática da educação em saúde no ambiente } \\
\text { hospitalar }\end{array}$ & Qualitativo \\
\hline $\begin{array}{l}2013 \\
\text { Arábia } \\
\text { Sáudita }\end{array}$ & Medicina & $\begin{array}{l}\text { Impact of indoor education on the lifestyles } \\
\text { of patients with chronic disease in secon- } \\
\text { dary hospital in Qassim, Kingdom of Saudi } \\
\text { Arabia }\end{array}$ & $\begin{array}{l}\text { Determinar o impacto da educação interna } \\
\text { em um hospital secundário em Qassim, Rei- } \\
\text { no de Unido }\end{array}$ & Corte \\
\hline $\begin{array}{l}2013 \\
\text { Japão }\end{array}$ & $\begin{array}{l}\text { Enfermagem } \\
\text { e Medicina }\end{array}$ & $\begin{array}{l}\text { The effects of naacessibility-enchanced- } \\
\text { multimídia informational educational pro- } \\
\text { grammeem reducing anxiety and increasing } \\
\text { satisfaction of patients undergoing cardiac } \\
\text { catheterization }\end{array}$ & $\begin{array}{l}\text { Avaliar a eficácia de um programa educacio- } \\
\text { nal informativo multimídia acessível para } \\
\text { reduzir a ansiedade e aumentar a satisfação } \\
\text { com as informações e materiais recebidos por } \\
\text { pacientes submetidos a cateterismo cardíaco }\end{array}$ & Corte \\
\hline $\begin{array}{l}2016 \\
\text { Reino } \\
\text { Unido }\end{array}$ & Enfermagem & $\begin{array}{l}\text { Exploring the potential impact of health } \\
\text { promotion videos as a low cost intervention } \\
\text { to reduce health inequalities: pilot before } \\
\text { and after study on Bangladeshis in inner-city } \\
\text { London }\end{array}$ & $\begin{array}{l}\text { Estudar o uso e a eficácia da promoção de } \\
\text { saúde por vídeo para uma doença evitável }\end{array}$ & Transversal \\
\hline $\begin{array}{l}2016 \\
\text { Brasil }\end{array}$ & Enfermagem & $\begin{array}{l}\text { Knowledge and meaning of cardiac cathe- } \\
\text { terization from the perspective of cardiac } \\
\text { patients }\end{array}$ & $\begin{array}{l}\text { Descrever o conhecimento e significado do } \\
\text { cateterismo cardíaco para pacientes cardio- } \\
\text { patas }\end{array}$ & Qualitativo \\
\hline
\end{tabular}

Font: Dados da pesquisa.

\section{DISCUSSÃO}

A apresentação da discussão será através das categorias que emergiram por meio da análise das informações: déficit de conhecimento dos pacientes sobre cineangiocoronariografia e a construção do conhecimento por meio de diferentes abordagens metodológicas. 


\section{DÉFICIT DE CONHECIMENTO DOS PACIENTES SOBRE CINEANGIOCORONARIOGRÁFICA}

A cineangiocoronariografia/cateterismo cardíaco é um exame diagnóstico intervencionista, realizado em larga escala no laboratório de hemodinâmica, normalmente de forma eletiva (previamente agendados), porém também podem ter ocorrências emergenciais como no caso de um infarto agudo do miocárdio. Este exame tornou-se um procedimento comum para pacientes portadores da doença arterial coronariana (DAC), por fornecer informações detalhadas sobre a anatomia cardiovascular, auxiliando no prognóstico e na melhor terapêutica ${ }^{3-5,13}$.

Apesar de este exame apresentar demanda significativa, percebe-se que o conhecimento sobre o mesmo, por parte dos pacientes, ainda mostra-se limitado e publicações sobre esta temática são escassas ${ }^{5,13}$. Em estudo realizado por Castro et al. ${ }^{6}$ sobre o conhecimento dos pacientes acerca do cateterismo cardíaco, com uma mostra de 30 pacientes de um hospital público no Estado do Maranhão, observou-se que $70 \%$ dos entrevistados mostraram pouco conhecimento sobre o procedimento. Há pacientes que confundem o cateterismo cardíaco diagnóstico com a angioplastia, procedimento realizado quando, durante o exame, é diagnosticado lesão na artéria coronária passível de ser resolvida com a utilização de "balão" ou "stent". Outros relataram medo e ansiedade, principalmente pelas expectativas geradas diante do desconhecido. Este fato evidencia a carência e lacunas nas informações passadas para os pacientes em relação ao exame, bem como na percepção dos profissionais quanto às reais necessidades, preocupações e inseguranças desses pacientes.

Outros estudos salientam que a equipe de saúde atuante nos serviços de hemodinâmica, muitas vezes tem sua atenção voltada para as complicações pós-exame, em detrimento das fases anteriores do mesmo, o que também intensifica os sentimentos de angústia, ansiedade e medo o que pode inclusive levar piora a evolução clínica ${ }^{3,5}$.

No entanto, pacientes que foram submetidos previamente ao cateterismo cardíaco, apresentam-se menos ansiosos e temerosos, pois já sabem como será realizado e qual o motivo do exame. Salientando assim como o conhecimento prévio pode ajudar para uma boa evolução $0^{6}$. Tal fato é corroborado nos estudos de Rigon \& Neves 9 e Júnior et $\mathrm{al}^{7}$ quando elucidam que, quanto maior o grau de conhecimento sobre seu problema de saúde, os pacientes tendem a apresentar mais segurança e confiança para o autocuidado e melhor adesão ao tratamento.

A educação em saúde no ambiente hospitalar, a qual deveria ser responsável por transformar o sujeito/ paciente em agente ativo de sua aprendizagem, necessita ser modificada, pois vem sendo ministrada em uma prática de orientações pontuais, prescritiva e focada na doença, tornando-a reducionista e fundamentada em ensinamentos impostos, fazendo dos pacientes simples objetos, passivos de intervenções. Um serviço de alta complexidade como da hemodinâmica, por ser um campo de trabalho relativamente novo para a enfermagem, requer do profissional enfermeiro constantes atualizações podendo por vezes tornar sua atuação centrada nos aspectos biológicos e tecnicista em detrimento à relação interpessoal profissional paciente ${ }^{5-6}$.

Em um estudo de coorte retrospectivo realizado na Arábia Saudita, com 169 pacientes portadores de doenças crônicas, evidenciou-se a educação no ambiente hospitalar como ferramenta importante. Resultados positivos e efetivos dependem da frequência com que são realizadas as orientações durante o período de internação, as habilidades de relacionamento interpessoal, adaptação do linguajar e empatia durante o atendimento. Os autores consideram a educação familiar como outra carência no contexto da educação em saúde uma vez que a abordagem adaptada quando realizada de forma efetiva para pacientes e familiares, melhora a aderência, aumenta a satisfação, ameniza a ansiedade e contribui para prevenção de complicações das doenças crônicas, melhorando a qualidade de vida dos indivíduos ${ }^{14}$.

Assim torna-se indispensável a elaboração de estratégias de educação em saúde que visem à aproximação e à interação entre a equipe profissional e os pacientes. Estratégias estas que aperfeiçoem o fluxo de informações durante o período pré, trans e pós-cateterismo, dispondo de recursos como multimídia, folhetos explicativos, espaço para esclarecimentos verbais e apresentação dos passos do exame $e^{7,13-15}$. 
A CONSTRUÇÃO DO CONHECIMENTO POR MEIO DE DIFERENTES ABORDAGENS METODOLÓGICAS

$\mathrm{O}$ uso de materiais educativos é considerado um recurso que possui a tarefa de alinhar e inserir na sociedade normas, padróes de comportamentos e mudança de hábitos ${ }^{16-18}$. Pode-se encontrá-los sob as mais variadas formas, como folder, animações audiovisuais, simulações, questionários de avaliações, entre outros ${ }^{17-18}$. Porém, por si só não garantem a aprendizagem, pois há fatores que interferem neste processo como experiências pessoais e a própria intenção do sujeito em entender o significado do conteúdo passado.

Um dos métodos mais utilizados na educação em saúde, atualmente, são os materiais impressos que usam uma lógica transmissional, um modelo unilinear de comunicação, o qual restringe os espaços para negociação de sentidos, filiando-se ao paradigma biomédico, o que acarreta em certa impessoalidade, por não ser o ideal há necessidade de se extinguir tal prática. ${ }^{17,18}$

Com os avanços tecnológicos, a internet também se tornou uma ferramenta importante na disseminação de conhecimento e apresenta informações sobre os mais diversos temas relacionados à saúde. Muitas informações a respeito da cineangiocoronariografia estão disponíveis nos meios de comunicação, mas frequentemente, os pacientes chegam para a sua realização com dúvidas e preocupações sobre o procedimento e suas possíveis intercorrências ${ }^{5,6}$.

Varo e Modi $^{13}$ corroboram com tal elucidação quando, em sua pesquisa realizada no Reino Unido, avaliaram informações disponíveis on-line sobre $\mathrm{o}$ tema angioplastia, nos sites google, yaboo e bing, que correspondem a $94 \%$ da circulação de pesquisa, constataram-se que a maioria dos usuários clicam na primeira página e raramente olham para as próximas. Ainda, referiram que as informações disponibilizadas normalmente apresentam qualidade variável, pois, a maioria mostrou-se desatualizada ou com literatura médica muito complexa para o leitor. Há ainda, produções acadêmicas, sites corporativos patrocinados, muitas vezes promovem determinado dispositivo e fóruns on-line onde pacientes compartilham experiências de doenças. Porém, não há regulamentação do conteúdo, qualquer pessoa com o desejo de produzir um site relacionado à saúde pode fazê-lo.

Neste contexto, a preocupação é que pacientes cardiopatas não têm, em sua grande maioria, conhecimento para avaliar criticamente a qualidade dos dados que acessam, necessitando assim que os serviços de saúde, inclusive o laboratório de hemodinâmica, elaborem estratégias para esclarecimento de dúvidas $\mathrm{e}$ informações sobre os procedimentos para os pacientes.

Em estudo ${ }^{16}$ piloto realizado em Bangladeshi com pacientes que chegavam para realização do cateterismo cardíaco no laboratório de hemodinâmica, foi aplicado um questionário contendo 15 questões, sendo cinco questões sobre conhecimento básico de fisiologia cardíaca e doença arterial coronariana e dez questões sobre fatores de risco modificáveis para desenvolvimento de doença cardiovascular e cuidados pré e pós-exame. Após o preenchimento deste questionário os pacientes assistiam a um vídeo educacional elaborado em concordância com as diretrizes cardiovasculares, logo após o questionário era reaplicado. O número médio de respostas corretas no pré-teste foi de 9,38 e após a visualização a média de acertos evoluiu para 11,67, mostrando que em curto prazo este método educacional melhora significativamente o conhecimento sobre a doença, com impacto positivo na segurança e compreensão do exame.

Visto que a grande demanda diária dificulta o processo de orientação individual à aplicação do recurso audiovisual além de apresentar baixo custo e fácil manuseio, é uma ferramenta útil e mais abrangente no que diz respeito a interagir com pessoas de todos os estágios intelectuais e de todas as faixas etárias.

No Rio Grande do Sul/Brasil, estudo similar foi realizado por Torrano et $\mathrm{al}^{3}{ }^{3}$ com 94 pacientes, os quais realizariam cateterismo cardíaco diagnóstico eletivo ambulatorial, os grupos foram separados em grupo controle e grupo intervenção. O grupo controle respondia o questionário sobre o exame e, após, assistia a filme. $\mathrm{O}$ grupo intervenção assistia ao filme e após respondia o questionário. O resultado comprovou que os indivíduos que assistiam ao vídeo antes de responder o questionário (grupo intervenção) adquiriam maior conhecimento sobre a doença e o exame, além de apresentar satisfação a partir das informações recebidas. 
Para Wu et al. ${ }^{15}$ apenas um método educacional é pouco para amenizar ansiedade e impactar efetivamente a vida dos pacientes. Em seu estudo randomizado, realizado no Japão, com o objetivo de avaliar a eficácia de um programa educacional informativo de multimídia na redução da ansiedade e aumento da satisfação com as informações e materiais recebidos por pacientes submetidos a cateterismo cardíaco, observou-se a necessidade de associar os métodos educacionais. No referido estudo a estratégia metodológica com maior impacto e efetividade para redução da ansiedade nos pacientes foi o que se associou à educação convencional, à utilização de multimídia e apoio multiprofissional.

O profissional de saúde, ao elaborar o material educativo deve considerar as questões sociodemográficas, cultural e o saber do paciente, bem como a criação de espaço para reflexão e compartilhamento de conhecimentos entre profissionais e pacientes é o meio mais enriquecedor e traz maiores benefícios, tornando-se assim imprescindível a associação dos recursos tecnológicos com prática da escuta qualificada, melhorando a comunicação e o relacionamento interpessoal $^{17,18}$.

\section{CONCLUSÃO}

Os estudos descritos nesta pesquisa demonstraram a eficácia e a importância da saúde educacional quando realizada com embasamento científico e ético, com uma metodologia baseada na construção compartilhada do conhecimento, por meio do diálogo, considerando e valorizando as vivências do paciente. Esta educação, quando associada aos recursos impressos e audiovisuais melhora significativamente o conhecimento sobre o procedimento, a doença arterial coronariana e cuidados pré e pós-cateterismo, impactando positivamente na saúde dos usuários.

É importante ressaltar que é primordial a publicação de estudos científicos originais sobre educação em saúde no serviço de hemodinâmica que possibilitem a troca de experiências entre os profissionais e enriqueça a dinâmica de trabalho, o que ainda não é uma realidade no Brasil.

\section{REFERÊNCIAS}

1. Ministério da saúde (BR). Secretaria Executiva Datasus. Informações de Saúde. Mortalidade e informações epidemiológicas [Internet]. 2012. [acesso em 2016 Nov. 10]. Disponível em: http://tabnet.datasus.gov.br/ cgi/idb2012/matriz.htm

2. Simão AF, Precoma DB, Andrade JP, Correa Filho H, Saraiva JFK, Oliveira GMM et al. SBC I Diretriz Brasileira de Prevenção Cardiovascular. Arq. Bras. Cardiol. [Internet]. 2013 [acesso em 2016 Nov. 10]; 101(6, supl. 2):1-78. Disponível em: http:// publicacoes.cardiol.br/consenso/2013/Diretriz_ Prevencao_Cardiovascular.pdf

3. Torrano, SK. Veiga, VB, Goldmeier, S, Azzolin, K. Explanatory digital video disc with patients undergoing diagnostic cardiac catheterization. Rev Lat Am Enfermagem [Internet]. 2011 [acesso em 2016 Nov. 30]; 19(4): 888-93. Disponível em: http://dx.doi. org/10.1590/\$0104-11692011000400006

4. Piegas LS, Timerman A, Feitosa GS, Nicolau JC, Mattos LAP, Andrade MD, et al. SBC/N. Diretriz Brasileira de tratamento de infarto agudo do miocárdio com supra desnivelamento do segmento ST. Arq. Bras. Cardiol. [Internet]. 2015 [aceso em 2016 Dez. 03]; 105(2): 135. Disponível em: http://publicacoes.cardiol.br/2014/ img/pockets/POCKETBOOK_2015_Interativa.pdf

5. Costa GR, Cardoso SB, Sousa LL, Soares TR, Ferreira AKA, Lima FF. Atuação do enfermeiro no serviço de hemodinâmica: uma revisão integrativa. Rev Interdisciplinar. 2014;7(3):157-64.

6. Castro YTBO, Rolim ILTP, Silva ACO, Silva LDCS. Knowledge and meaning of cardiac catheterization from the perspective of cardiac patients. Rev Rene [Internet]. 2016[acesso em 2016 Dez. 03];17(1):2935. Disponível em: http://www.revistarene.ufc.br/ revista/index.php/revista/article/view/2150

7. Nogueira-Junior C, Gama BMBM, Teixeira MS, ArreguySena C. Educação em enfermagem: desafio diário para cuidar com excelência uma análise da vivência de uma equipe. Rev Enferm. Cent. Oeste Mineiro 
[Internet]. 2011 [acesso em 2016 Nov. 30]; 1(4): 546-59. Disponível em: http://www.seer.ufsj.edu.br/ index.php/recom/article/viewFile/149/243

8. Figueira AB, Amestoy DC, Tristão FS, Trindade LL, Correa VA. Visão do enfermeiro frente à prática da educação em saúde no ambiente hospitalar. Ver Cogitare Enferm [Internet]. 2013 [acesso em 2016 Nov. 30]; 18(2): 310-6. Disponível em: http://revistas. ufpr.br/cogitare/article/view/32580

9. Rigon, AG, Neves ET. Educação em saúde e a atuação de enfermagem no contexto de unidades de internação hospitalar: o que tem sido ou há para ser dito? Texto Contexto Enferm, Florianópolis. 2011; 20(4): 812-7.

10. Salci MA, Maceno P, Rozza SG, Silva DMGV, Boehs AE, Heidemann ITSB. Educação em saúde e suas perspectivas teóricas: algumas reflexões. Texto Contexto Enferm. [Internet]. 2013 [acesso em 2016 Dez 03]; 22(1): 224-30. Disponível em: http://www. scielo.br/pdf/tce/v22n1/pt_27.pdf

11. Ganong LH. Integrative review of nursing research. Res Nursing Health. [Internet] 1987 [acesso em 2016 Nov. 30]; 10(1). Disponível em: https://www.ncbi. nlm.nih.gov/pubmed/3644366

12. Galvão CM, Paula CC, Padoin SMM. Revisão integrativa como ferramenta para tomada de decisão na prática em saúde. In: Lacerda MR, Costenaro, RGS, organizadores. Metodologias da pesquisa para a enfermagem e saúde: da teoria à prática. Lacerda MR, Costenaro, RGS. Porto Alegre: Moriá, 2015. p. 51-76.

13. Varo KMA, Modi BN. Coronary Angioplasty and the Internet: what can patients searching online expect to find? JournalofinterventionalCardiology [Internet]. 2012 [acesso em 2016 Dez. 03]; 25(5): 476-81. Disponível em: https://www.ncbi.nlm.nih. gov/pubmed/22672356

14. Midhet FM, Mohaimeed MD. Impact of indoor education on the lifetyles of patients with chronic diasease in a secondary hospital in Qassim, Kingdom of Saudi Arabia. Journal of Taibah University Med. Sciences [Internet]. 2013 [acesso em 2016 Nov. 30];
8(1): 44-49. Disponível: http://www.sciencedirect. com/science/article/pii/S165836121300005X

15. Wu KL, Chen SR, Ko WC, Kuo SY, Chen PL, Su HF, Chang WY. The effectiveness of an accessibility-enhanced multimedia informational educational programme in reducing anxiety and increasing satisfaction of patients undergoing cardiac catheterization. Journal of Clinical Nursing [Internet]. 2013 [acesso em 2016 Nov. 30]; 23: 2063-2073. Disponível em: http://onlinelibrary. wiley.com/doi/10.1111/jocn.12469/abstract

16. Latif S, Ahmed I, Amin MS, Syed I, Ahmed N. Exploring the potential impact of health promotion videos as a low cost intervention to reduce health inequalities: pilot before and after study on Bangladeshis in innercity London. J. of Primary Care. 2016; 8(4): 66-71.

17. Freitas FV, Filho LAR. Modelos de comunicação e uso de impressos na educação em saúde: uma pesquisa bibliográfica. Rev Interface, 2011; 15(36): 243-255.

18. Trindade CS, Dahmer A, Reppold CT. Objetos de aprendizagem: uma revisão integrativa na área da saúde. Journal de Health Informatics [Internet]. 2014 [acesso em 2016 Dez 03]; 6(1): 20-29. Disponível em: http://www.jhi-sbis.saude.ws/ojs-jhi/index.php/jhisbis/article/view/300

Recebido em:

Aceito em: 